\title{
Organofilização de argilas bentoníticas com tensoativos não- iônicos visando seu uso em fluidos de perfuração base óleo
}

\section{(Nonionic surfactants organophilization bentonite clays aiming their use in oil base drilling fluids)}

\author{
I. A. Silva', J. M. R. Costa , H. S. Ferreira ${ }^{2}$, R. R. Menezes $^{2}$, G. A. Neves ${ }^{1}$, H. C. Ferreira ${ }^{1}$ \\ ${ }^{\prime}$ Unidade Acadêmica de Engenharia de Materiais, UFCG \\ ${ }^{2}$ Departamento de Engenharia de Materiais, Centro de Tecnologia, UFPB \\ romualdomenezes@gmail.com,gelmires@dema.ufcg.edu.br,heber@dema.ufcg.edu.br
}

\begin{abstract}
Resumo
O uso de tensoativos não iônicos vem substituindo os tradicionais tensoativos iônicos dentre outros motivos pelo seu elevado potencial de resistência a degradação térmica. Assim, este trabalho tem por objetivo o desenvolvimento de argilas organofílicas através da incorporação de tensoativos não iônicos visando seu uso em fluidos de perfuração de poços de petróleo base óleo. Argilas bentoníticas natural, industrializadas e organofilizadas foram caracterizadas por análise química, difração de raios $\mathrm{X}$ e análises térmicas. Após a organofilização foi realizado o inchamento de Foster, visando a escolha dos tensoativos mais adequados aos meios líquidos orgânicos dispersantes: éster, diesel e parafina. Com as dispersões obtidas foram determinadas as propriedades reológicas através de viscosidades aparente e plástica. Os resultados evidenciaram que as argilas organofilicas apresentaram um aumento do espaçamento interlamelar e que as dispersões apresentaram propriedades reológicas dentro das especificações da Petrobrás, para uso como argilas organofílicas em fluidos de perfuração em base óleo.

Palavras-chave: organofilização, argilas bentoníticas, tensoativos não iônicos.
\end{abstract}

\begin{abstract}
The nonionic surfactants use is replacing the traditional ionic surfactants among other reasons because of its high resistance to thermal degradation potential. This work aims the organoclays development through the nonionic surfactants incorporation in order to oil base drilling fluids use. The natural bentonite clay, and industrialized organophilizated were characterized by chemical analysis, X-ray diffraction and thermal analysis. After, the clay organophilization was performed by Foster swelling, seeking the most appropriate choice of surfactants to the liquid media organic dispersants, ester, paraffin and diesel. With the obtained dispersions were determined by plastic and apparent viscosities the rheological properties. The results showed that organoclays increase in the interlayer spacing and that the dispersions showed rheological properties within the Petrobras specifications for use in oil base drilling fluids.
\end{abstract}

Keywords: organophilization, bentonite clays, nonionic surfactants.

\section{INTRODUÇÃO}

As bentonitas são argilas extremamente versáteis e apresentam uma extensa gama de usos industriais [1]. As bentonitas, apesar da conceituação geológica clássica, modernamente podem ser consideradas como qualquer argila constituída por argilominerais do grupo da esmectita que apresentem propriedades similares as bentonitas clássicas [2]. Um maior detalhamento de diferenças relativas às terminologias e definições de bentonitas, esmectitas e montmorilonita podem ser encontradas [1-3]. A versatilidade das bentonitas resultam de aspectos relativos à sua formação geológica que conduzem a um material que se enquadra perfeitamente no conceito "taylor made", permitindo grandes modificações planejadas em sua microestrutura, o que permite obter propriedades específicas e faz com que esses materiais sejam as argilas com maior quantidade de usos industriais. Em parte estas propriedades resultam de uma microestrutura extremamente peculiar, com áreas especificas teóricas da ordem de $800 \mathrm{~m}^{2} / \mathrm{g}$, constituída por lamelas extremamente finas com cerca de $10 \AA$, e da habilidade de sua microestrutura interagir com variada gama de produtos orgânicos e inorgânicos [1]. Dentro desta ótica as bentonitas reagem com os tensoativos iônicos, anfóteros e não iônicos através de uma série de mecanismos que estão descritos detalhadamente nas referências $[1,3,4]$, formando um grupo especial de materiais denominados de argilas organofilicas. Argilas organofílicas são argilas que contém moléculas orgânicas intercaladas entre suas camadas estruturais ou adsorvidas nas superfícies. A inserção de moléculas orgânicas faz com que ocorram expansões entre os planos $d_{001}$ da argila e muda sua natureza hidrofílica para hidrofóbica ou organofílica. As argilas organofílicas possuem uma extensa gama de aplicações industriais [2,5], podendo-se destacar 
seus usos em: fluidos de perfuração de poços de petróleo base óleo; indústrias de fundição de metais; lubrificantes; tintas; adesivos; cosméticos; amaciantes; condicionadores de cabelo; germicidas, etc. [6-8]. Recentemente, as argilas organofílicas também estão sendo utilizadas como cargas na obtenção de nanocompósitos visando promover mudanças nas propriedades mecânicas, físicas e químicas de matrizes poliméricas [9]. A modificação superficial de argilas esmectíticas é uma área que tem recebido bastante atenção porque permite ampliar os horizontes de aplicação das argilas, gerando novos materiais e novas aplicações [1].

Os fluidos de perfuração de poços de petróleo mais tradicionais são os à base de água. No entanto, em virtude das exigências relativas a perfurações cada vez mais profundas e as condições de perfuração em alto mar ("off shore") tornase imperativo o uso de fluidos de perfuração especiais, com especial destaque para os fluidos base óleo contendo argilas organofilicas [10-13].

Para composição destes fluidos as argilas organofilicas inicialmente eram obtidas com sais quaternários de amônio, estes sais, entretanto, apresentam algumas limitações devido à baixa estabilidade térmica e sua elevada resistência a degradação quando dispostos no meio ambiente. Visando a solução desses problemas foram introduzidos os tensoativos não iônicos. Ao contrário dos tensoativos iônicos cujo mecanismo de interação com as bentonitas ocorre fundamentalmente através da troca de cátions, os tensoativos não iônicos interagem através de fenômenos de adsorção. Devido a não existência de íons ou grupos funcionais de quimiosorção nos tensoativos não iônicos, pontes de hidrogênio podem ser consideradas a principal força motriz para a adsorção dos surfactantes não iônicos $[1,4]$.

Verificou-se que é possível obter elevados espaçamentos basais e quantidade de carbono orgânico em bentonitas organofílicas com a utilização de tensoativos não iônicos. Ademais, esses tensoativos apresentam menor toxidade e maior potencial de biodegradação que os sais quaternários de amônio e as argilas organofílicas produzidas apresentam maior estabilidade química que as obtidas com surfactantes catiônicos [14]. Por outro lado, há uma grande demanda em pesquisas para desenvolvimento e aprimoramento de fluidos de perfuração base orgânica, frente os desafios atuais enfrentados pela indústria petrolífera nacional e a necessidade de redução na importação de argilas organofílicas. Assim, este trabalho tem por objetivo o desenvolvimento de argilas organofílicas através da adição de tensoativos não iônicos visando seu uso em fluídos de perfuração de poços de petróleo base óleo.

\section{MATERIAIS E MÉTODOS}

No desenvolvimento desta pesquisa foram utilizados os seguintes materiais: cloisite $\mathrm{Na}^{ \pm} \underline{\mathbb{R}}$ - argila bentonítica industrializada importada, naturalmente sódica, proveniente da empresa "Southern Clay", Gonzáles, TX, EUA; Brasgel

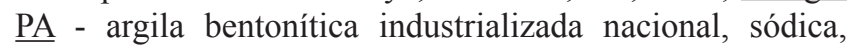

proveniente da empresa Bentonit União Nordeste-BUN, Campina Grande, PB; argila Chocolate - argila bentonítica natural, policatiônica, proveniente da Mina Bravo, Boa Vista, PB; Ultramina 20 - amina etoxilada TA20®, com $100 \%$ de matéria ativa, gentilmente cedida pelo fabricante Oxiteno S/A Ind. Com., S. Paulo, SP; Ultramina 50 - amina etoxilada TA50®, com $100 \%$ de matéria ativa, gentilmente cedida pelo fabricante Oxiteno S/A Ind. Com., S. Paulo, SP; Imidazolina oleica vegetal - IMI, produzida em escala experimental, também cedida pela Oxiteno S/A Ind. Com.; Meios líquidos orgânicos dispersantes - foram utilizados três líquidos orgânicos como meios dispersantes: 1) óleo diesel tipo $\mathrm{D}$, produzido especialmente para a utilização em motores de embarcações marítimos apresenta-se com cadeia carbônica de 6 a 30 átomos sendo composto por hidrocarbonetos parafínicos, oleofínicos e aromáticos; 2) éster, que possui estrutura química complexa sendo derivado da reação entre vários ácidos graxos, mono e di funcionais com cadeia entre três a doze carbonos e álcoois mono, di e tri funcionais e 3) parafina, composta de uma mistura de hidrocarbonetos (principalmente alcanos) de alto peso molecular, formada predominantemente, por 13 átomos de carbono e organizada de forma linear, não possuindo uma composição química definida, utilizados para fluidos de perfuração.

A caracterização das amostras de argilas natural, industrializadas e organofiliadas foi efetuada por meio das seguintes técnicas: análise química por fluorescência de raios X ( EDX 720 Shimadzu); difração de raios X (D6000 Shimadzu), com radiação Cuka $(40 \mathrm{kV} / 30 \mathrm{~mA})$ e rotação do goniômetro de $2^{\circ} /$ min e passo $0,02^{\circ}$, com faixa de $1,5^{\circ}$ a $30^{\circ}$ para a amostra pura e com faixa de $2^{\circ}$ a $10^{\circ}$ para as amostras organofilizadas); análise termogravimétrica (ATG) e análise térmica diferencial (ATD) (sistema de análises térmicas TA $60 \mathrm{H}$ Shimadzu), com taxa de aquecimento $12,5^{\circ} \mathrm{C} / \mathrm{min}$ sob atmosfera de nitrogênio. A temperatura máxima para ambos os casos foi $1000^{\circ} \mathrm{C}$ e o padrão utilizado na ATD e TG foi óxido de alumínio $\left(\mathrm{Al}_{2} \mathrm{O}_{3}\right)$ calcinado. Para todas as análises as amostras apresentam granulometria inferior a peneira ABNT 200 (0,074 mm).

A Tabela I apresenta as legendas associadas aos tensoativos e argilas.

Para o processo de organofilização foram utilizados 3,615 $\mathrm{L}$ de água deionizada, onde adicionou-se $150 \mathrm{~g}$ da argila, agitou-se por $20 \mathrm{~min}$ e foi adicionado o tensoativo não iônico (Tabela I) onde foi ajustado o $\mathrm{pH}$ até 7,0, com uma solução de $\mathrm{HCl}$ a $2 \mathrm{~N}$; após o ajuste do $\mathrm{pH}$, a agitação foi mantida por mais $20 \mathrm{~min}$; realizou-se a filtração e em seguida o sistema foi levado a estufa a $60^{\circ} \mathrm{C}$, onde permaneceu entre $24 \mathrm{~h}$ e 96 h; por fim a argila oganofilizada foi beneficiada em moinho de bolas e passado em peneira ABNT $200(0,074 \mathrm{~mm})$. A concentração de tensoativo utilizado na organofilização foi de $20 \%$ e de $30 \%$, sendo traduzidas em massa por $30 \mathrm{~g}$ e $45 \mathrm{~g}$, respectivamente. Após organofilização foram determinadas as medidas do inchamento de Foster [15], sendo realizadas utilizando os meios líquidos orgânicos dispersantes anteriormente mencionados e as classificações consideradas 
Tabela I - Legendas de associação entre os tensoativos e porcentagens, combinados com as argilas Closite $\mathrm{Na}^{+}$, Brasgel PA e Chocolate utilizadas.

[Table I - Legends relating surfactants and percentages, combined with Closite Na+, Brasgel PA and Chocolate clays used.]

\begin{tabular}{ccc}
\hline $\begin{array}{c}\text { Argila Closite Na}{ }^{+} \text {, tensoativo e teor } \\
\text { incorporado (\% mássica) }\end{array}$ & $\begin{array}{c}\text { Argila Brasgel PA, tensoativo e teor } \\
\text { incorporado (\% mássica) }\end{array}$ & $\begin{array}{c}\text { Argila Chocolate, tensoativo e teor } \\
\text { incorporado (\% mássica) }\end{array}$ \\
\hline CL-Na-TA20-20\% & BR-TA20-20\% & CH-TA20-20\% \\
CL-Na-TA20-30\% & BR-TA20-30\% & CH-TA20-30\% \\
CL-Na-TA50-20\% & BR-TA50-20\% & CH-TA50-20\% \\
CL-Na-TA50-30\% & BR-TA50-30\% & CH-TA50-30\% \\
CL-Na-IMI-20\% & BR-IMI-20\% & CH-IMI-20\% \\
CL-Na-IMI-30\% & BR-IMI-30\% & CH-IMI-30\% \\
\hline
\end{tabular}

para o inchamento foram: valores iguais ou inferiores a 2 $\mathrm{mL} / \mathrm{g}$ foram considerados como "não inchamento"; de 3 a $5 \mathrm{~mL} / \mathrm{g}$ como "inchamento baixo"; de 6 a $8 \mathrm{~mL} / \mathrm{g}$ como "inchamento médio" e acima de $8 \mathrm{~mL} / \mathrm{g}$ como "inchamento alto". Este método mostra a compatibilidade do meio estudado com a argila organofílica obtida [7]. Os fluidos de perfuração base orgânica foram preparados da seguinte forma: em um copo do agitador Hamilton Beach N5000 adicionou-se $336 \mathrm{~mL}$ do meio líquido orgânico e $84 \mathrm{~mL}$ de uma solução saturada de $\mathrm{NaCl}(0,395 \mathrm{~g} / \mathrm{mL})$ agitando-se por 5 min. Esta emulsão é chamada de lama base. Depois foi adicionada a argila organofílica nas quantidades de $2,4 \mathrm{~g}$, $6,0 \mathrm{~g}, 9,6 \mathrm{~g}$ e 13,2 g de argila organofílica/420 mL de lama base, respectivamente, e a agitação foi mantida por $20 \mathrm{~min}$. Depois de preparado, o fluido foi depositado em um "roller over" (estufa rotativa) aquecida a $66^{\circ} \mathrm{C}$, permanecendo no seu interior por $16 \mathrm{~h}$ para ocorrer o envelhecimento. Em seguida foram determinadas a viscosidades aparente (VA) e a viscosidade plástica (VP) de acordo com a normatização da Petrobrás [16]. Deve-se mencionar que as normas da Petrobrás [16] foram elaboradas para o meio dispersante óleo diesel marítimo (tipo D). Assim, para os meios líquidos dispersantes ésteres e parafinas foi utilizada a normatização da Petrobrás aplicada a óleo diesel por inexistência de norma específica.

\section{RESULTADOS E DISCUSSÃO}

Na Tabela II estão apresentadas as composições químicas das argilas Cloisite $\mathrm{Na}^{+}$, Brasgel PA e Chocolate. Observouse que o teor de $\mathrm{SiO}_{2}$ é similar em todas as amostras de argilas; no entanto, para o teor $\mathrm{Al}_{2} \mathrm{O}_{3}$ tem-se que as argilas chocolate e Brasgel PA apresentaram teores similares enquanto a Cloisite $\mathrm{Na}^{+}$apresentou um teor significativamente mais elevado. Isso evidencia um maior teor de fração argila nesse material.

Para o teor de $\mathrm{Fe}_{2} \mathrm{O}_{3}$, observaram-se grandes diferenças entre as argilas, as argilas Chocolate e Brasgel apresentaram elevado teor, já a Cloisite $\mathrm{Na}^{+}$apresentou um teor significativamente menor, essa redução, deve-se provavelmente ao processo beneficiamento da amostra Cloisite $\mathrm{Na}^{+}$. As argilas Chocolate e Cloisite $\mathrm{Na}^{+}$apresentaram baixo teor de $\mathrm{CaO}$, já a Brasgel apresentou um teor mais elevando evidenciando a origem geológica policatiônica da argila. Os teores de $\mathrm{MgO}$ apresentaram-se similares evidenciando a presença do magnésio em todas as amostras. Para o teor de $\mathrm{Na}_{2} \mathrm{O}$, observa-se que a argila Chocolate, de natureza policatiônica, não apresentou o sódio na sua composição, já as argilas Brasgel e Cloisite $\mathrm{Na}^{+}$, a primeira tornada sódica industrialmente e a segunda naturalmente sódica, apresentaram o sódio em sua composição. $\mathrm{O} \mathrm{K}_{2} \mathrm{O}$ e outros óxidos apresentaram-se em baixos teores e presentes em todas as amostras de forma similar. A perda ao rubro (PR) apresentou valores similares em todas as amostras. De maneira global verificou-se que as argilas possuem composição química típica de argilas bentoníticas $[2,17$, $18]$.

Na Fig. 1 estão apresentados os resultados de difração de raios $\mathrm{X}$ das argilas bentoníticas Observou-se para argila Chocolate a presença do argilomineral esmectítico, caracterizado pela distância interplanar de $15,56 \AA$ e 4,45 Å, presença de quartzo, caracterizado pelas distâncias

Tabela II - Composição química das argilas Cloisite $\mathrm{Na}^{+}$, Brasgel PA e Chocolate.

[Table II - Chemical composition of Cloisite $\mathrm{Na}^{+}$, Brasgel PA and Chocolate clays.]

\begin{tabular}{cccccccccc}
\hline \multirow{2}{*}{ Amostras } & \multicolumn{10}{c}{ Composição química (\%) } \\
\cline { 2 - 10 } & $\mathrm{SiO}_{2}$ & $\mathrm{Al}_{2} \mathrm{O}_{3}$ & $\mathrm{Fe}_{2} \mathrm{O}_{3}$ & $\mathrm{CaO}$ & $\mathrm{MgO}$ & $\mathrm{Na}_{2} \mathrm{O}$ & $\mathrm{K}_{2} \mathrm{O}$ & Outros $^{\mathrm{a}}$ & $\mathrm{PR}^{\mathrm{b}}$ \\
\hline Chocolate & 59,77 & 17,31 & 10,37 & 0,30 & 2,21 & - & 0,21 & 1,07 & 8,72 \\
Brasgel PA & 62,33 & 16,45 & 7,76 & 1,26 & 2,09 & 1,65 & 0,52 & 1,18 & 6,61 \\
Cloisite $\mathrm{Na}^{+}$ & 60,14 & 23,34 & 4,10 & 0,40 & 2,05 & 2,37 & 0,03 & 0,71 & 6,82 \\
\hline
\end{tabular}

${ }^{a}$ Outros óxidos, ${ }^{b}$ Perda ao Rubro determinada por calcinação em amostras secas a $110^{\circ} \mathrm{C}$. 
interplanares de 4,26 $\AA$ e 3,34 A. Para argila Brasgel PA, verificou-se a presença do argilomineral esmectítico, caracterizado pela distância interplanar de 12,86 $\AA$ e 4,43 $\AA$ e a presença de quartzo, caracterizado pelas distâncias interplanares 4,67 $\AA$ e 3,34 $\AA$. Para argila Cloisite $\mathrm{Na}^{+}$, verificou-se a presença do argilomineral esmectítico, caracterizada pela distância interplanar $12,16 \AA, 4,45 \AA \mathrm{e}$ $3,12 \AA$. As amostras foram tratadas com etileno glicol para confirmação da presença do argilomineral esmectítico. De maneira global verifica-se que as argilas possuem difratogramas típicos das argilas bentoníticas $[2,17,18]$.

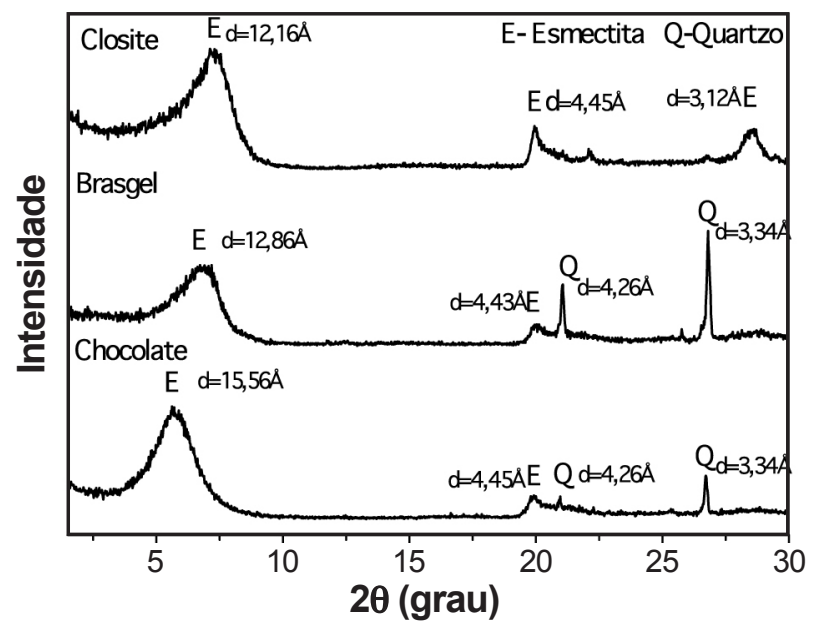

Figura 1: Difratogramas de raios $\mathrm{X}$ das argilas Cloisite $\mathrm{Na}^{+}$, Brasgel PA e Chocolate.

[Figure 1: X-ray diffraction patterns of Cloisite $\mathrm{Na}^{+}$, Brasgel PA and Chocolate clays.]

A Fig. 2 apresenta as curvas de análises termogravimétricas e térmicas diferenciais das argilas bentoníticas: Closite $\mathrm{Na}^{+}$(a), Brasgel PA (b) e Chocolate (c). Analisando-se as curvas de ATD da argila Cloisite $\mathrm{Na}^{+}$(Fig. 2a) verificaramse as seguintes transformações térmicas: pico endotérmico de grande intensidade a $159^{\circ} \mathrm{C}$, característico da presença de água livre e adsorvida; pico endotérmico de pequena intensidade a $710{ }^{\circ} \mathrm{C}$, característica da presença de hidroxilas da folha octaédrica, típico das esmectitas pobre em ferro; pico endotérmico de pequena intensidade a $880^{\circ} \mathrm{C}$, característico da destruição do retículo cristalino, pico exotérmico a $910^{\circ} \mathrm{C}$, característica da nucleação da mulita, com liberação de quartzo beta. Analisando-se as curvas de TG, foram observados os eventos característicos da perda de água e perda de hidroxilas e uma perda total de massa de cerca de $17 \%$.

Com base nas curvas de ATD da argila Brasgel PA (Fig. 2b) verificou-se as seguintes transformações térmicas: grande pico endotérmico a $155^{\circ} \mathrm{C}$, característica da presença de água livre e adsorvida; pico a $552{ }^{\circ} \mathrm{C}$, característico da presença de hidroxilas da folha octaédrica; pico endotérmico a $797{ }^{\circ} \mathrm{C}$, característica da provável presença do carbonato de magnésio e pico de pequena intensidade a $880{ }^{\circ} \mathrm{C}$, característico da destruição do retículo cristalino e pico exotérmico a $899^{\circ} \mathrm{C}$ da nucleação da mulita com liberação de quartzo beta. Analisando as curvas de TG, foram observados os eventos característicos da perda de água, perda de hidroxilas e uma perda total de massa de
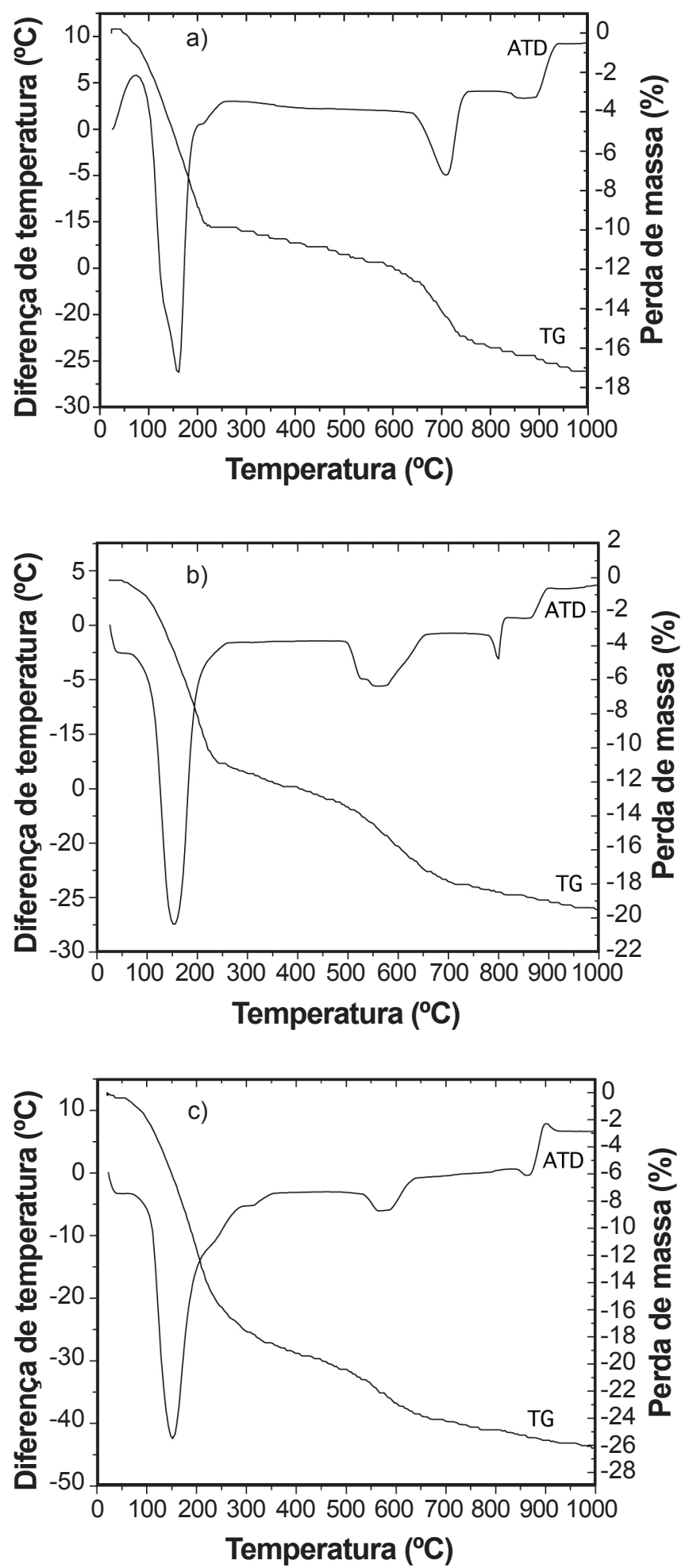

Figura 2: Curvas de análise térmica das argilas: a) Cloisite $\mathrm{Na}^{+}, \mathrm{b}$ ) Brasgel PA e c) Chocolate.

[Figure 2: Thermal analysis curvesof: a) Cloisite $\mathrm{Na}^{+}$, b) Brasgel $P A$ and c) Chocolate.] 
cerca de 19\%. As curvas de ATD da argila chocolate (Fig. 2c) apresentaram as seguintes transformações térmicas: grande pico endotérmico a $150{ }^{\circ} \mathrm{C}$, característica da presença de água livre e adsorvida; pico endotérmico a $572{ }^{\circ} \mathrm{C}$, característico da presença de hidroxilas da folha octaédrica; pico endotérmico a $864{ }^{\circ} \mathrm{C}$, característico da destruição do retículo cristalino e pico exotérmico a $903{ }^{\circ} \mathrm{C}$ da nucleação da mulita com liberação de quartzo beta. Analisando-se as curvas de TG, foram observados os eventos característicos da perda de água e perda de hidroxilas e uma perda total de massa de cerca de $26 \%$. Numa análise conjunta observa-se, a redução nas faixas de temperaturas referente à presença de hidroxilas, devido à presença de esmectitas ricas em ferro, como visto na Tabela II, sendo as demais características de argilominerais esmectíticos [2, 17, 18].

Na Fig. 3 estão apresentados os difratogramas de raios $\mathrm{X}$ da argila Closite $\mathrm{Na}^{+}$com tensoativos não iônicos TA20, TA50 e IMI, a 20\% e 30\%. Observaram-se distâncias interplanares basais semelhantes, porém, há um aumento na distância interplanar basal original da Closite $\mathrm{Na}^{+}$pura de 12 $\AA$, para $56,2 \AA$ com o tensoativo TA20 a $20 \%$ e o maior valor encontrado que foi de 58,1 A para o tensoativo TA50 a $20 \%$.

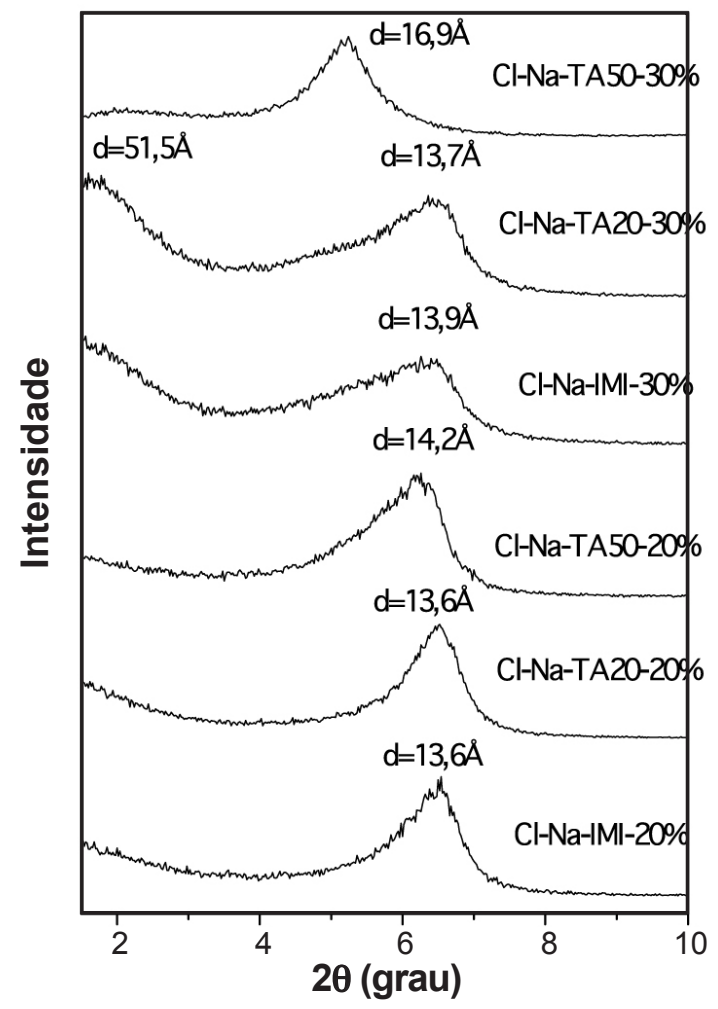

Figura 3: Difratogramas de raios $\mathrm{X}$ da argila Closite $\mathrm{Na}^{+}$ organofilizada com os tensoativos TA 20, TA50 e IMI a 20\% e $30 \%$. [Figure 3: X-ray diffraction patterns of Closite $\mathrm{Na}^{+}$clay with $20 \%$ and $30 \%$ of surfactants TA 20, TA50 and IMI.]

Nos difratogramas da Fig. 3 observaram-se distâncias interplanares basais semelhantes; porém, houve um elevado aumento da distância interplanar basal original da Closite $\mathrm{Na}^{+}$pura de $12 \AA \AA$ e o menor valor encontrado que é de $57,3 \AA$ com IMI a $30 \%$ e o maior valor encontrado foi de
$59,2 \AA$ com o tensoativo TA20 a $30 \%$; mostrando-se que houve eficácia no processo de intercalação dos tensoativos mencionados na argila, aumentando à distância interplanar basal.

Na Fig. 4 estão apresentados os difratogramas de raios $\mathrm{X}$ da argila Brasgel PA com tensoativos não iônicos TA20, TA50 e IMI, a $20 \%$ e 30\%. Observaram-se distâncias interplanares basais semelhantes; porém, houve um elevado aumento da distância interplanar basal original da Brasgel PA pura, de $12 \AA$ para o menor valor encontrado que foi de $56,2 \AA$ com tensoativo TA50 a $20 \%$ e para o maior valor foi de $60,0 \AA$ com IMI a $20 \%$.

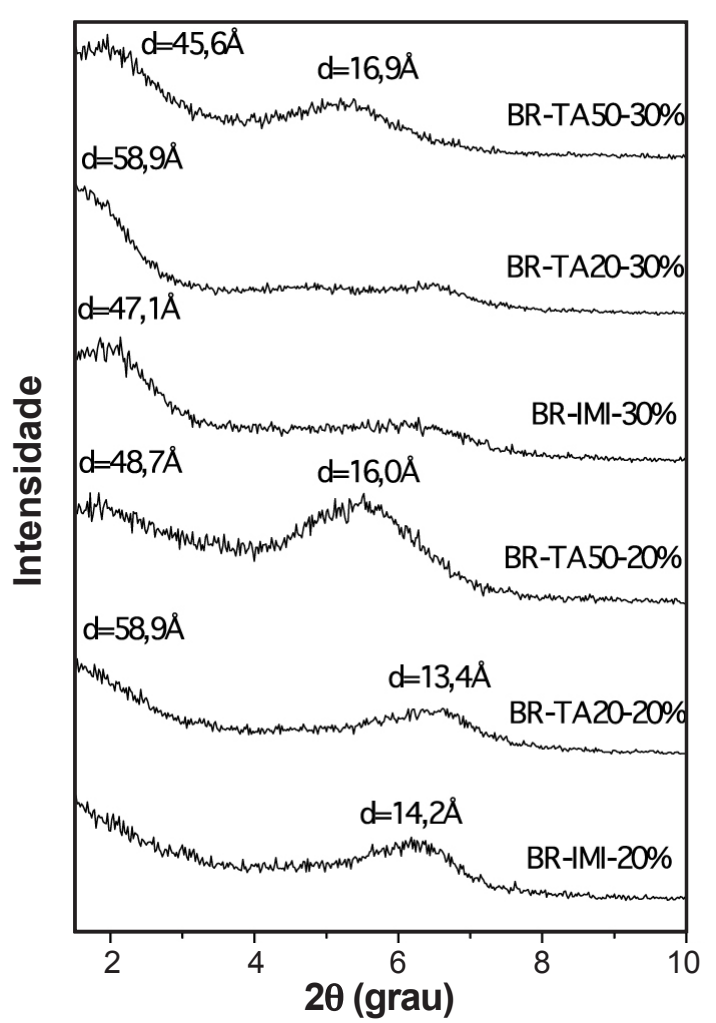

Figura 4: Difratogramas de raios $\mathrm{X}$ da argila Brasgel PA com os tensoativos TA20, TA50 e IMI a $20 \%$ e $30 \%$.

[Figure 4: X-ray diffraction patterns of Brasgel PA clay with $20 \%$ and $30 \%$ of surfactant TA20, TA50 and IMI.]

Observaram-se distâncias interplanares basais semelhantes, porém, houve um elevado aumento no valor da distância interplanar basal original da Brasgel PA pura, de $12 \AA$ para o menor valor encontrado que foi de $56,2 \AA$ com tensoativo TA50 a $30 \%$ e para o maior valor que foi de 58,1 Å com o tensoativo TA20 a $30 \%$ e com IMI a $30 \%$; mostrando-se que houve eficácia do processo de intercalação dos tensoativos mencionados na argila, aumentando de forma à distância interplanar basal.

Na Fig. 5 estão apresentados os difratogramas de raios $\mathrm{X}$ da argila Chocolate com tensoativos não iônicos TA20, TA50 e IMI, a 20 e $30 \%$. Analisando os difratogramas da Fig. 5 da argila Chocolate com os tensoativos TA20, TA50 e IMI a $20 \%$, observaram-se distâncias interplanares basais 
semelhantes, porém, houve um elevado aumento no valor da distância interplanar basal original da argila Chocolate pura, de $17 \AA$ para o menor valor encontrado que foi de $55,2 \AA$ com tensoativo TA20 a $20 \%$ e para o maior valor que foi de 57,3 Å com tensoativo IMI a $20 \%$.

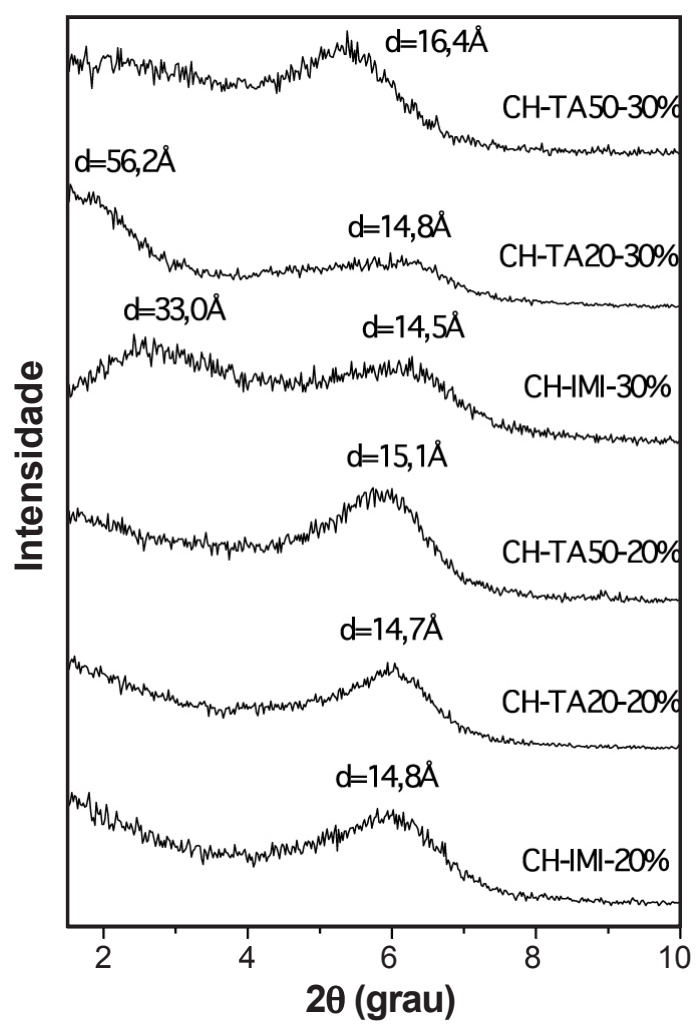

Figura 5: Difratogramas de raios $\mathrm{X}$ da argila Chocolate organofilizada com os tensoativos TA20, TA50 e IMI a 20\% e 30\%. [Figure 5: X-ray diffraction patterns of Chocolate clayorganofilized with $20 \%$ and $30 \%$ of surfactant TA20, TA50 and IMI.]

Observaram-se distâncias interplanares basais semelhantes, porém, houve um elevado aumento no valor da distância interplanar basal original da argila Chocolate pura, de 17 $\AA$ para o menor valor encontrado que foi de $57,3 \AA$ com tensoativos TA20 a $30 \%$ e TA50 a $30 \%$ e para o maior valor que foi de 58,07 $\AA$ com tensoativo IMI a $30 \%$, mostrandose que houve eficácia no processo de intercalação dos tensoativos mencionados na argila, aumentando à distância interplanar basal.

$\mathrm{Na}$ Tabela III estão apresentados os valores da perda de massa das argilas Closite $\mathrm{Na}^{+}$, Bragel PA e Chocolate organofilizada e a porcentagem de tensoativo adsorvido.

Analisando os valores da Tabela III para argila Closite $\mathrm{Na}^{+}$, verificou-se que a perda de massa corresponde à perda de água livre e adsorvida, hidroxilas e tensoativos, sendo o valor máximo de 33,68\% correspondente à argila incorporada com TA50 com 30\%. O valor mínimo foi de $26,64 \%$ para a argila incorporada com tensoativo IMI com 20\%. Para argila Brasgel PA, verificou-se que a perda de massa máxima foi de $35,52 \%$ correspondente à argila incorporada com o tensoativo TA50 com $30 \%$, e
Tabela III - Perda de massa das argilas Closite $\mathrm{Na}^{+}$, Bragel PA e Chocolate organofilizada e a porcentagem de tensoativo adsorvido.

[Table III - Loss of mass of organophilized Closite $\mathrm{Na}^{+}$, Brasgel PA and Chocolate clays (w/w) and surfactant adsorbed percentage $(w / w)$.]

\begin{tabular}{ccc}
\hline $\begin{array}{c}\text { Argila }- \\
\text { Tipo de tensoativo }- \\
\text { Teor incorporado }\end{array}$ & $\begin{array}{c}\text { Perda de } \\
\text { massa } \\
(\%)\end{array}$ & $\begin{array}{c}\text { Tensoativo } \\
\text { adsorvido } \\
(\%)\end{array}$ \\
\hline CL-TA20-20\% & 27,79 & 17,61 \\
CL-TA20-30\% & 32,75 & 22,57 \\
CL-TA50-20\% & 26,74 & 16,56 \\
CL-TA50-30\% & 33,68 & 23,50 \\
CL-IMI-20\% & 26,64 & 16,46 \\
CL-IMI-30\% & 33,10 & 22,92 \\
BR-TA20-20\% & 28,34 & 15,95 \\
BR-TA20-30\% & 34,10 & 21,71 \\
BR-TA50-20\% & 30,05 & 17,66 \\
BR-TA50-30\% & 35,52 & 23,13 \\
BR-IMI-20\% & 29,56 & 17,17 \\
BR-IMI-30\% & 33,77 & 21,38 \\
CH-TA20-20\% & 28,16 & 10,88 \\
CH-TA20-30\% & 34,98 & 17,7 \\
CH-TA50-20\% & 27,96 & 10,41 \\
CH-TA50-30\% & 33,09 & 15,81 \\
CH-IMI-20\% & 28,22 & 10,94 \\
CH-IMI-30\% & 33,15 & 15,87 \\
\hline
\end{tabular}

o valor mínimo foi de $28,34 \%$ para a argila incorporada com tensoativo TA20 com 20\%. Para argila Chocolate, verificou-se que a perda de massa máxima foi de 34,98\% correspondente à argila incorporada com o tensoativo TA20 com $30 \%$ e o valor mínimo foi de $27,96 \%$ para a argila incorporada com tensoativo TA50 com 20\%. Comparandose de maneira geral os resultados de tensoativos adsorvidos obtidos com o valor teórico a $20 \%$ incorporado à argila, verificou-se que os valores de tensoativos adsorvidos em média foram de $16,87 \%, 16,92 \%$ e $10,74 \%$ para as argilas Closite $\mathrm{Na}^{+}$, Bragel PA e Chocolate respectivamente, sendo inferiores ao valor de perda de massa teórica que foram de $22,35 \%, 22,17 \%$ e $24,0 \%$, respectivamente. Para as incorporações a $30 \%$ à argila, verificou-se que os valores de tensoativos adsorvidos em média foram de 22,99\%, 22,07\% e $16,46 \%$ para as argilas Closite $\mathrm{Na}^{+}$Bragel PA e Chocolate respectivamente, também inferiores aos valores de perda de massa teórica que foram de $28,32 \%, 28,16 \%$ e $29,78 \%$, respectivamente, confirmando a teoria de que houve a eficaz incorporação dos tensoativos na argila, aumentando assim a sua perda de massa.

$\mathrm{Na}$ Tabela IV estão apresentados os resultados do inchamento de Foster das argilas Closite $\mathrm{Na}^{+}$, Bragel PA e Chocolate organofilizada com tensoativos e porcentagens 
Tabela IV - Inchamento de Foster das argilas Closite $\mathrm{Na}^{+}$, Bragel PA e Chocolate organofiliza nos meios orgânicos: éster, parafina e óleo diesel.

[Table IV - Foster swelling of organophilized Closite $\mathrm{Na}^{+}$, Bragel PA and Chocolate clays in organic media: ester, paraffin and diesel oil.]

\begin{tabular}{ccccccc}
\hline $\begin{array}{c}\text { Argila - Tipo de } \\
\text { tensoativo - } \\
\text { Teor incorporado }\end{array}$ & $24 \mathrm{~h}$ & $48 \mathrm{~h}$ & $24 \mathrm{~h}$ & $48 \mathrm{~h}$ & $24 \mathrm{~h}$ & $48 \mathrm{~h}$ \\
\hline CL_Na_TA20_20\% & 2,5 & 3,5 & 1,3 & 1,75 & 2,10 & 2,10 \\
CL_Na_TA20_30\% & 4,0 & 4,75 & 1,75 & 1,75 & 1,30 & 1,75 \\
CL_Na_TA50_20\% & 2,5 & 3,5 & 1,75 & 1,75 & 2,10 & 2,45 \\
CL_Na_TA50_30\% & 3,5 & 4,75 & 1,75 & 1,75 & 3,0 & 4,0 \\
CL_Na_IMI_20\% & 1,0 & 2,0 & 1,05 & 1,30 & 1,25 & 1,30 \\
CL_Na_IMI30\% & 1,0 & 2,0 & 1,05 & 1,30 & 1,25 & 1,30 \\
BR_TA20_20\% & 6,0 & 6,0 & 1,3 & 1,5 & 3,0 & 4,0 \\
BR_TA20_30\% & 6,0 & 7,0 & 1,5 & 1,75 & 4,0 & 5,0 \\
BR_TA50_20\% & 6,0 & 7,0 & 1,3 & 1,5 & 3,0 & 4,0 \\
BR_TA50_30\% & 7,0 & 8,0 & 1,5 & 1,75 & 4,0 & 6,0 \\
BR_IMI_20\% & 2,5 & 2,5 & 1,0 & 1,5 & 2,0 & 2,5 \\
BR_IMI_30\% & 2,0 & 2,5 & 1,0 & 1,5 & 2,5 & 2,5 \\
CH_TA20_20\% & 6,0 & 8,0 & 1,3 & 1,75 & 3,0 & 4,0 \\
CH_TA20_30\% & 8,0 & 9,0 & 1,4 & 1,8 & 5,0 & 6,0 \\
CH_TA50_20\% & 6,0 & 8,0 & 1,3 & 1,75 & 3,0 & 4,0 \\
CH_TA50_30\% & 6,0 & 8,0 & 1,5 & 1,8 & 5,0 & 6,0 \\
CH_IMI_20\% & 2,0 & 2,5 & 1,05 & 1,30 & 2,0 & 2,5 \\
CH_IMI_30\% & 2,0 & 2,5 & 1,05 & 1,30 & 2,5 & 3,0 \\
\hline
\end{tabular}

nos meios dispersantes orgânicos: éster, parafina e diesel.

Verificou-se que os melhores resultados obtidos foram para o meio dispersante orgânico éster e o segundo o diesel. Em relação aos tensoativos, verificou-se que o TA20 e o TA50 com $20 \%$ e $30 \%$ de tensoativo para argila organofílica Chocolate foram os que obtiveram melhores resultados com valores superiores a $8 \mathrm{~mL} / \mathrm{g}$ indicando "inchamento alto", comprovando uma melhor compatibilidade com os meios ésteres, óleo diesel e parafina. Para a Bragel PA incorporadas com tensoativos TA20 e TA50 os valores encontrados estão na faixa entre $6 \mathrm{~mL} / \mathrm{g}$ e $8 \mathrm{~mL} / \mathrm{g}$ sendo classificados como inchamento médio, e os demais são classificados como inchamento baixo ou não incharam. Na Tabela $\mathrm{V}$ estão apresentado os resultados da viscosidade aparente e plástica no meio dispersante orgânico éster com a argila Closite $\mathrm{Na}^{+}$ organofílica a $20 \%$ e $30 \%$ dos tensoativos TA20, TA50 e IMI. Analisando os valores das viscosidades aparente (VA) e plástica (VP) para o meio dispersante orgânico éster e a argila Closite $\mathrm{Na}^{+}$organofilizada com TA20, TA50 e IMI a $20 \%$ e $30 \%$, contidos na Tabela V, verificou-se que os melhores resultados foram encontrados na argila incorporada com tensoativo TA50 a $30 \%$, com valores máximos de viscosidades aparente e plástica de $17,0 \mathrm{cP}$ e 12 $\mathrm{cP}$, respectivamente. Estes resultados estão de acordo com os previstos pelo inchamento de Foster [14]. Comparando estes valores com as especificações da Petrobrás [15], verificou-se que os resultados satisfazem os requisitos das normas para uso em fluido de perfuração base óleo.

$\mathrm{Na}$ Tabela VI estão apresentado os resultados das viscosidades aparente e plástica no meio dispersante orgânico éster com a argila Brasgel PA organofílica a $20 \%$ e $30 \%$ dos tensoativos TA20, TA50 e IMI. Analisando os valores das viscosidades aparente (VA) e plástica (VP) para o meio dispersante orgânico éster e a argila Bragel PA organofilizada com TA20, TA50 e IMI a 20\% e 30\%, contidos na Tabela VI, verificou-se que os melhores resultados foram encontrados na argila incorporada com tensoativo TA50 com $30 \%$, sendo os valores máximos de viscosidades aparente e plástica de $16,5 \mathrm{cP}$ e $12 \mathrm{cP}$, respectivamente. Estes resultados estão de acordo com os previstos pelo inchamento de Foster [15]. Comparando esses valores com as especificações da Petrobrás [16], verificou-se que estes resultados satisfazem todos requisitos das normas para uso em fluido de perfuração base óleo.

Na Tabela VII estão apresentado os resultados das viscosidades aparente e plástica no meio dispersante orgânico éster com a argila Chocolate organofílica a 20\% e $30 \%$ dos tensoativos TA20, TA50 e IMI. Com base nos valores contidos na Tabela VII, verificou-se que os melhores resultados foram encontrados na argila incorporada 
Tabela V - Viscosidades aparentes (VA) e plásticas (VP) no meio líquido orgânico dispersante éster com a argila Closite $\mathrm{Na}^{+}$organofilizada com TA20, TA50 e IMI a $20 \%$ e $30 \%$.

[Table V-Apparent (VA) and plastic (VP) viscosities of the organic liquid ester containing Closite $\mathrm{Na}^{+}$clay organophilized with $20 \%$ and $30 \%$ of TA20, TA50 and IMI.]

\begin{tabular}{|c|c|c|c|c|c|}
\hline Teor de tensoativo de $20 \%$ & $\mathrm{VA}(\mathrm{cP})$ & $\mathrm{VP}(\mathrm{cP})$ & Teor de tensoativo de $30 \%$ & $\mathrm{VA}(\mathrm{cP})$ & $\mathrm{VP}(\mathrm{cP})$ \\
\hline CL-TA20-20\% ${ }^{a}$ & 8,0 & 8,0 & CL-TA20-30\% ${ }^{\mathrm{a}}$ & 9,0 & 8,0 \\
\hline CL-TA20-20\% ${ }^{\mathrm{a}}$ & 8,0 & 7,0 & CL-TA20-30\% ${ }^{a}$ & 11,5 & 10,0 \\
\hline CL-TA20-20\% ${ }^{a}$ & 10,0 & 9,0 & CL-TA20-30\% ${ }^{a}$ & 9,5 & 9,0 \\
\hline CL-TA20-20\% ${ }^{a}$ & 10,0 & 9,0 & CL-TA20-30\% ${ }^{a}$ & 10,5 & 10,0 \\
\hline CL-TA50-20\% ${ }^{a}$ & 9,0 & 8,0 & CL-TA50-30\% ${ }^{a}$ & 8,5 & 8,0 \\
\hline CL-TA50-20\% ${ }^{a}$ & 8,5 & 8,0 & CL-TA50-30\% ${ }^{a}$ & 9,5 & 9,0 \\
\hline CL-TA50-20\% ${ }^{a}$ & 8,5 & 8,0 & CL-TA50-30\% ${ }^{a}$ & 12,0 & 9,0 \\
\hline CL-TA50-20\% ${ }^{a}$ & 8,5 & 8,0 & CL-TA50-30\% ${ }^{a}$ & 17,0 & 12,0 \\
\hline CL-IMI- $20 \%{ }^{a}$ & 10,0 & 10,0 & CL-IMI- $30 \%{ }^{\mathrm{a}}$ & 9,5 & 9,0 \\
\hline CL-IMI- $20 \%{ }^{a}$ & 9,5 & 10,0 & CL-IMI- $30 \%{ }^{a}$ & 9,0 & 9,0 \\
\hline CL-IMI- $20 \%{ }^{a}$ & 9,5 & 9,0 & CL-IMI-30\% ${ }^{\mathrm{a}}$ & 9,5 & 10,0 \\
\hline CL-IMI- $20 \%{ }^{a}$ & 9,5 & 10,0 & CL-IMI-30\% ${ }^{\mathrm{a}}$ & 10,0 & 10,0 \\
\hline
\end{tabular}

${ }^{a}$ Teores de argila de 2,4; 6,0; 9,6; e 13,2 correspondentes aos requisitos mínimos de viscosidade aparente para fluidos de perfuração base óleo estabelecidos pela Petrobrás [16]

Tabela VI - Viscosidade aparente (VA) e Viscosidade plástica no meio líquido orgânico dispersante éster com a argila Brasgel PA organofilizada com TA20, TA50 e IMI a 20\% e 30\%.

[Table VI - Apparent (VA) and plastic (VP) viscosities of the organic liquid ester containing Brasgel PA clay organophilized with $20 \%$ and $30 \%$ of TA20, TA50 and IMI.]

\begin{tabular}{|c|c|c|c|c|c|}
\hline Teor de tensoativo de $20 \%$ & $\mathrm{VA}(\mathrm{cP})$ & $\mathrm{VP}(\mathrm{cP})$ & Teor de tensoativo de $30 \%$ & $\mathrm{VA}(\mathrm{cP})$ & $\mathrm{VP}(\mathrm{cP})$ \\
\hline BR-TA20-20\% ${ }^{\text {a }}$ & 4,5 & 6,5 & BR-TA20-30\% ${ }^{a}$ & 5,25 & 6,5 \\
\hline BR-TA20-20\% ${ }^{\text {a }}$ & 5,0 & 6,0 & BR-TA20-30\% ${ }^{a}$ & 7,0 & 7,0 \\
\hline BR-TA20-20\% ${ }^{\text {a }}$ & 6,5 & 6,5 & BR-TA20-30\% ${ }^{a}$ & 7,0 & 6,5 \\
\hline BR-TA20-20\% ${ }^{a}$ & 7,75 & 6,0 & BR-TA20-30\% ${ }^{\text {a }}$ & 8,0 & 6,0 \\
\hline BR-TA $50-20 \%^{a}$ & 6,0 & 7,0 & BR-TA50-30\% ${ }^{a}$ & 6,5 & 8,0 \\
\hline BR-TA50-20\% ${ }^{\text {a }}$ & 8,0 & 8,5 & BR-TA50-30\% ${ }^{a}$ & 8,5 & 9,0 \\
\hline BR-TA50-20\% ${ }^{\text {a }}$ & 10,0 & 9,0 & BR-TA50-30\% ${ }^{a}$ & 11,5 & 9,0 \\
\hline BR-TA50-20\% ${ }^{\text {a }}$ & 14,5 & 10,0 & BR-TA50-30\% ${ }^{\text {a }}$ & 16,5 & 12,0 \\
\hline BR-IMI-20\% ${ }^{\mathrm{a}}$ & 5,5 & 7,5 & BR-IMI- $30 \%{ }^{a}$ & 4,75 & 5,5 \\
\hline BR-IMI-20\% ${ }^{\text {a }}$ & 5,5 & 7,0 & BR-IMI- $30 \%{ }^{a}$ & 5,0 & 5,5 \\
\hline BR-IMI-20\% ${ }^{a}$ & 6,0 & 7,0 & BR-IMI- $30 \%{ }^{a}$ & 5,5 & 5,5 \\
\hline BR-IMI-20\% ${ }^{\mathrm{a}}$ & 6,5 & 7,0 & BR-IMI-30\% ${ }^{\mathrm{a}}$ & 5,5 & 5,5 \\
\hline
\end{tabular}

com tensoativo TA50 a 30\%, sendo os valores máximos de viscosidades aparente e plástica de $17,0 \mathrm{cP}$ e $12 \mathrm{cP}$, respectivamente. Estes resultados estão de acordo com os previstos pelo inchamento de Foster [15]. Comparando estes valores com as especificações da Petrobrás [16], verificouse que os resultados com tensoativo TA50 com 30\% de incorporação satisfazem todos os requisitos das normas para uso em fluido de perfuração base óleo.

$\mathrm{Na}$ Tabela VIII estão apresentados os resultados das viscosidades aparente e plástica no meio dispersante orgânico óleo diesel com a argila Closite $\mathrm{Na}^{+}$organofílica a $20 \%$ e $30 \%$ dos tensoativos TA20, TA50 e IMI. Verificouse que os melhores resultados foram encontrados na argila incorporada com tensoativo TA50 a $30 \%$, com valores máximos de viscosidades aparente e plástica de $16,5 \mathrm{cP}$ e 11 $\mathrm{cP}$, respectivamente. Estes resultados estão de acordo com os previstos pelo inchamento de Foster [15]. Comparando estes valores com as especificações da Petrobrás [16], verificouse que os resultados com o tensoativo TA50 com 30\% de incorporação satisfazem os requisitos das normas para uso em fluido de perfuração base óleo.

$\mathrm{Na}$ Tabela IX estão apresentados os resultados das 
Tabela VII - Viscosidade aparente (VA) e viscosidade plástica (VP) no meio líquido orgânico dispersante éster com a argila Chocolate organofilizada com os tensoativos TA20, TA50 e IMI com 20\% e 30\%. [Table VII - Apparent (VA) and plastic (VP) viscosities of the organic liquid ester containing Chocolate clay organophilized with $20 \%$ and $30 \%$ of TA20, TA50 and IMI.]

\begin{tabular}{|c|c|c|c|c|c|}
\hline Teor de tensoativo de $20 \%$ & $\begin{array}{l}\mathrm{VA} \\
(\mathrm{cP})\end{array}$ & $\begin{array}{l}\mathrm{VP} \\
(\mathrm{cP})\end{array}$ & Teor de tensoativo de $30 \%$ & $\begin{array}{c}\mathrm{VA} \\
\text { (cP) }\end{array}$ & $\begin{array}{c}\mathrm{VP} \\
\text { (cP) }\end{array}$ \\
\hline CH-TA20-20\% ${ }^{\text {a }}$ & 6,0 & 9,5 & CH-TA20-30\% ${ }^{a}$ & 8,0 & 8,0 \\
\hline CH-TA20-20\% ${ }^{\text {a }}$ & 6,0 & 9,0 & CH-TA20-30\% ${ }^{a}$ & 8,0 & 8,0 \\
\hline CH-TA20-20\% ${ }^{\text {a }}$ & 11,5 & 11,5 & CH-TA20-30\% ${ }^{a}$ & 10,0 & 10,0 \\
\hline CH-TA20-20\% ${ }^{\text {a }}$ & 10,5 & 10,0 & CH-TA20-30\% ${ }^{\text {a }}$ & 11,0 & 10,0 \\
\hline CH-TA50-20\% ${ }^{\text {a }}$ & 9,0 & 10,0 & CH-TA50-30\% ${ }^{a}$ & 8,0 & 8,0 \\
\hline CH-TA50-20\% ${ }^{\text {a }}$ & 8,0 & 8,0 & CH-TA $50-30 \%^{a}$ & 9,0 & 10,0 \\
\hline CH-TA50-20\% ${ }^{\text {a }}$ & 10,5 & 11,0 & CH-TA50-30\% ${ }^{a}$ & 12,0 & 12,0 \\
\hline CH-TA50-20\% ${ }^{a}$ & 12,0 & 12,0 & CH-TA50-30\% ${ }^{a}$ & 17,0 & 5,0 \\
\hline CH-IMI- $20 \%{ }^{a}$ & 9,5 & 9,0 & CH-IMI- $30 \%{ }^{a}$ & 9,5 & 9,0 \\
\hline CH-IMI-20\% ${ }^{\text {a }}$ & 9,0 & 9,0 & CH-IMI- $30 \%{ }^{a}$ & 9,0 & 9,0 \\
\hline CH-IMI-20\% ${ }^{a}$ & 9,5 & 10,0 & CH-IMI-30\% ${ }^{a}$ & 10,0 & 10,0 \\
\hline CH-IMI-20\% ${ }^{\text {a }}$ & 10,0 & 10,0 & CH-IMI-30\% ${ }^{a}$ & 11,0 & 10,0 \\
\hline
\end{tabular}

*Teores de argila de 2,4; 6,0; 9,6; e 13,2 correspondentes aos requisitos mínimos de viscosidade aparente para fluidos de perfuração base óleo estabelecidos pela Petrobrás [16]

Tabela VIII - Viscosidade aparente (VA) e viscosidade plástica (VP) do meio líquido orgânico dispersante óleo diesel com a argila Closite $\mathrm{Na}^{+}$organofilizada com $20 \%$ e $30 \%$ dos tensoativos TA20, TA50 e IMI.

[Table VIII - Apparent (VA) and plastic (VP) viscosities of the organic liquid diesel oil containing Closite $\mathrm{Na}^{+}$clay organophilized with $20 \%$ and $30 \%$ of TA20, TA50 and IMI.]

\begin{tabular}{|c|c|c|c|c|c|}
\hline Teor de tensoativo de $20 \%$ & $\begin{array}{c}\text { VA } \\
\text { (cP) }\end{array}$ & $\begin{array}{c}\mathrm{VP} \\
\text { (cP) }\end{array}$ & Teor de tensoativo de $30 \%$ & $\begin{array}{l}\mathrm{VA} \\
\text { (cP) }\end{array}$ & $\begin{array}{l}\mathrm{VP} \\
\text { (cP) }\end{array}$ \\
\hline CL-TA20-20\% $\%^{\mathrm{a}}$ & 5,5 & 5,0 & CL-TA20-30\% ${ }^{a}$ & 6,0 & 5,5 \\
\hline CL-TA20-20\% ${ }^{a}$ & 6,0 & 5,5 & CL-TA20-30\% ${ }^{\text {a }}$ & 5,75 & 5,0 \\
\hline CL-TA20-20\% ${ }^{\text {a }}$ & 7,0 & 6,0 & CL-TA20-30\% ${ }^{a}$ & 6,75 & 6,0 \\
\hline CL-TA20-20\% ${ }^{\text {a }}$ & 6,5 & 5,5 & CL-TA20-30\% ${ }^{a}$ & 10,5 & 8,0 \\
\hline CL-TA50-20\% ${ }^{a}$ & 5,5 & 8,0 & CL-TA50-30\% ${ }^{a}$ & 6,5 & 8,0 \\
\hline CL-TA50-20\% ${ }^{\mathrm{a}}$ & 6,0 & 7,0 & CL-TA50-30\% ${ }^{a}$ & 8,5 & 8,0 \\
\hline CL-TA50-20\% ${ }^{\mathrm{a}}$ & 10,0 & 9,0 & CL-TA50-30\% ${ }^{a}$ & 11,5 & 10,0 \\
\hline CL-TA50-20\% ${ }^{\mathrm{a}}$ & 14,0 & 10,0 & CL-TA $50-30 \%^{a}$ & 16,5 & 11,0 \\
\hline CL-IMI- $20 \%{ }^{a}$ & 4,0 & 4,0 & CL-IMI-30\% ${ }^{\mathrm{a}}$ & 4,75 & 5,0 \\
\hline CL-IMI- $20 \%{ }^{a}$ & 4,75 & 5,5 & CL-IMI-30\% ${ }^{\text {a }}$ & 4,5 & 5,0 \\
\hline CL-IMI-20\% ${ }^{a}$ & 6,0 & 6,0 & CL-IMI-30\% ${ }^{a}$ & 6,5 & 7,0 \\
\hline CL-IMI- $20 \%{ }^{a}$ & 6,25 & 6,25 & CL-IMI-30\% ${ }^{\mathrm{a}}$ & 6,5 & 7,0 \\
\hline
\end{tabular}

a Teores de argila de 2,4; 6,0; 9,6; e 13,2 correspondentes aos requisitos mínimos de viscosidade aparente para fluidos de perfuração base óleo estabelecidos pela Petrobrás [16]

viscosidades aparente e plástica no meio dispersante orgânico diesel com a argila Brasgel PA organofílica a $20 \%$ e $30 \%$ dos tensoativos TA20, TA50 e IMI. Observouse que os melhores resultados foram encontrados na argila incorporada com tensoativo TA50 a $20 \%$ e $30 \%$, com valores máximos de viscosidades aparente e plástica de $17,5 \mathrm{cP}$ e $15 \mathrm{cP}$, respectivamente. Estes resultados estão de acordo com os previstos pelo inchamento de Foster [15]. Comparando esses valores com as especificações da Petrobrás [16], verificou-se que os resultados com tensoativo TA50 com $30 \%$ de incorporação satisfazem os requisitos das normas para uso em fluido de perfuração base óleo.

$\mathrm{Na}$ Tabela $\mathrm{X}$ estão apresentados os resultados das viscosidades aparente e plástica no meio dispersante 
Tabela IX - Viscosidade aparente (VA) e (VP) do meio líquido orgânico dispersante óleo diesel com a argila Brasgel PA organofilizada com 20\% e 30\% dos tensoativos TA20, TA50 e IMI.

[Table IX - Apparent (VA) and plastic (VP) viscosities of the organic liquid diesel oil containing Bragel PA clay organophilized with $20 \%$ and $30 \%$ of TA20, TA50 and IMI.]

\begin{tabular}{|c|c|c|c|c|c|}
\hline Teor de tensoativo de $20 \%$ & $\begin{array}{c}\mathrm{VA} \\
\text { (cP) }\end{array}$ & $\begin{array}{l}\mathrm{VP} \\
(\mathrm{cP})\end{array}$ & Teor de tensoativo de $30 \%$ & $\begin{array}{c}\mathrm{VA} \\
(\mathrm{cP})\end{array}$ & $\begin{array}{l}\mathrm{VP} \\
\text { (cP) }\end{array}$ \\
\hline BR-TA20-20\% $\%^{a}$ & 5,5 & 7,0 & BR-TA20-30\% ${ }^{a}$ & 5,75 & 7,5 \\
\hline BR-TA20-20\% ${ }^{\text {a }}$ & 5,75 & 6,0 & BR-TA20-30\% ${ }^{a}$ & 6,25 & 7,0 \\
\hline BR-TA20-20\% ${ }^{\text {a }}$ & 6,75 & 7,0 & BR-TA20-30\% ${ }^{a}$ & 9,25 & 12,5 \\
\hline BR-TA20-20\% ${ }^{\text {a }}$ & 7,0 & 7,0 & BR-TA20-30\% ${ }^{\text {a }}$ & 7,0 & 7,5 \\
\hline BR-TA50-20\% ${ }^{\text {a }}$ & 5,5 & 3,0 & BR-TA50-30\% ${ }^{\text {a }}$ & 6,5 & 5,5 \\
\hline BR-TA50-20\% ${ }^{\text {a }}$ & 6,0 & 8,0 & BR-TA $50-30 \%{ }^{a}$ & 8,5 & 8,0 \\
\hline BR-TA50-20\% ${ }^{a}$ & 6,5 & 7,0 & BR-TA50-30\%a & 12,0 & 10,0 \\
\hline BR-TA50-20\% ${ }^{\text {a }}$ & 7,0 & 7,5 & BR-TA50-30\% ${ }^{a}$ & 17,5 & 15,0 \\
\hline BR-IMI- $20 \%{ }^{a}$ & 3,5 & 4,5 & BR-IMI-30\% ${ }^{\mathrm{a}}$ & 4,0 & 5,0 \\
\hline BR-IMI-20\% ${ }^{\text {a }}$ & 5,0 & 5,5 & BR-IMI-30\% ${ }^{\mathrm{a}}$ & 5,25 & 6,0 \\
\hline BR-IMI-20\% ${ }^{a}$ & 5,25 & 6,0 & BR-IMI-30\% ${ }^{\mathrm{a}}$ & 5,75 & 6,5 \\
\hline BR-IMI-20\% ${ }^{\text {a }}$ & 5,5 & 6,0 & BR-IMI- $30 \%{ }^{\text {a }}$ & 6,0 & 6,0 \\
\hline
\end{tabular}

${ }^{a}$ Teores de argila de 2,4; 6,0; 9,6; e 13,2 correspondentes aos requisitos minimos de viscosidade aparente para fluidos de perfuração base óleo estabelecidos pela Petrobrás [16]

Tabela X - Viscosidade aparente (VA) e (VP) do meio líquido orgânico dispersante óleo diesel com a argila Chocolate organofilizada com $20 \%$ e $30 \%$ dos tensoativos TA20, TA50 e IMI.

[Table X - Apparent (VA) and plastic (VP) viscosities of the organic liquid diesel oil containing Chocolate clay organophilized with $20 \%$ and $30 \%$ of TA20, TA50 and IMI.]

\begin{tabular}{|c|c|c|c|c|c|}
\hline Teor de tensoativo de $20 \%$ & $\begin{array}{c}\mathrm{VA} \\
\text { (cP) }\end{array}$ & $\begin{array}{l}\mathrm{VP} \\
(\mathrm{cP})\end{array}$ & Teor de tensoativo de $30 \%$ & $\begin{array}{c}\mathrm{VA} \\
\text { (cP) }\end{array}$ & $\begin{array}{l}\mathrm{VP} \\
\text { (cP) }\end{array}$ \\
\hline CH-TA20-20\% ${ }^{\mathrm{a}}$ & 6,5 & 7,5 & CH-TA20-30\% ${ }^{a}$ & 6,25 & 6,5 \\
\hline CH-TA20-20\% ${ }^{a}$ & 6,0 & 6,5 & CH-TA20-30\% ${ }^{a}$ & 6,25 & 6,5 \\
\hline CH-TA20-20\% ${ }^{\text {a }}$ & 6,5 & 6,5 & CH-TA20-30\% ${ }^{a}$ & 7,25 & 7,0 \\
\hline CH-TA20-20\% ${ }^{a}$ & 7,0 & 7,0 & CH-TA20-30\% ${ }^{a}$ & 7,5 & 7,0 \\
\hline CH-TA50-20\% ${ }^{a}$ & 6,25 & 8,5 & CH-TA50-30\% ${ }^{a}$ & 6,0 & 8,0 \\
\hline CH-TA $50-20 \%{ }^{a}$ & 6,0 & 6,5 & CH-TA $50-30 \%{ }^{a}$ & 8,5 & 10,0 \\
\hline CH-TA50-20\% ${ }^{a}$ & 6,5 & 7,0 & CH-TA50-30\% ${ }^{a}$ & 12,5 & 13,0 \\
\hline CH-TA50-20\% ${ }^{a}$ & 7,0 & 7,0 & CH-TA50-30\% ${ }^{a}$ & 16,5 & 14,0 \\
\hline CH-IMI- $20 \%{ }^{a}$ & 10,0 & 10,0 & CH-IMI- $30 \%{ }^{a}$ & 9,5 & 9,0 \\
\hline CH-IMI-20\% ${ }^{\text {a }}$ & 9,5 & 10,0 & CH-IMI- $30 \%{ }^{\text {a }}$ & 9,0 & 9,0 \\
\hline CH-IMI- $20 \%{ }^{a}$ & 9,5 & 9,0 & CH-IMI- $30 \%{ }^{a}$ & 9,5 & 10,0 \\
\hline CH-IMI- $20 \%{ }^{\text {a }}$ & 9,5 & 10,0 & CH-IMI- $30 \%{ }^{a}$ & 10,0 & 10,0 \\
\hline
\end{tabular}

${ }^{a}$ Teores de argila de 2,4; 6,0; 9,6; e 13,2 correspondentes aos requisitos mínimos de viscosidade aparente para fluidos de perfuração base óleo estabelecidos pela Petrobrás [16]

orgânico óleo diesel com a argila Chocolate organofílica com incorporação de $20 \%$ e $30 \%$ dos tensoativos TA20, TA50 e IMI. Analisando os valores das viscosidades aparente (VA) e plástica (VP), observou-se que os melhores resultados foram encontrados na argila incorporada com tensoativo TA50 a $30 \%$, com valores máximos de viscosidades aparente e plástica de $16,5 \mathrm{cP}$ e 14,0 cP, respectivamente. Estes resultados estão de acordo com os previstos pelo inchamento de Foster [15].
Comparando estes valores com as especificações da Petrobrás [16], verificou-se que os resultados com o tensoativo TA50 incorporado com 30\% satisfaz os requisitos das normas para uso em fluido de perfuração base óleo.

Os fluidos obtidos com o meio dispersante parafina não se adequaram aos requisitos citados anteriormente, independente da argila utilizada e do tensoativo utilizado, por isso os seus resultados não foram apresentados. 


\section{CONCLUSÕES}

Após estudos com tensoativos não-iônicos no desenvolvimento de argilas organofílicas visando uso em fluidos de perfuração de poços de petróleo base óleo, concluiu-se que: os tensoativos estudados mostraram eficácia no processo de incorporação nas argilas, aumentando de forma notável o espaçamento interlamelar; nas análises termogravimétricas foi possível quantificar a incorporação dos tensoativos não iônicos nas argilas estudadas; nas análises de inchamento de Foster, foi possível observar que o meio dispersante éster apresentou uma melhor compatibilidade com as argilas organofílicas obtidas com os diversos tipos de tensoativos; os resultados das propriedades reologias evidenciam que o meio dispersante éster apresentou melhores resultados de viscosidade aparente (VA) seguido do óleo diesel, sendo o tensoativo TA50 com 30\% de incorporação o que atende as especificações da Petrobrás para uso em perfuração de poços de petróleo base óleo.

\section{REFERÊNCIAS}

[1] A. R. V. Silva, H. C. Ferreira, Rev. Eletr. Mater. Proc. 3, $2(2008) 26$.

[2] P. Souza Santos, Tecnologia de argilas, $3^{\text {a }}$ Ed., Vol. 3 Edgard Blücher, S. Paulo, SP (1992).

[3] F. Bergaya, B. K. G. Theng, G. Lagaly, Handbook of clay science, $1^{\text {st }}$ Ed., Developments in Clay Science 1, Elsevier Science, New York, EUA (2006).

[4]L.A.Ultracki, Clay-Containg Polymeric Nanocomposites, Ed. Rapra Technol. Ltd., UK, 1 (2004).
[5] S. Silva, P. E. Araújo, K. M. Ferreira, E. Canedo, L. Carvalho, C. M. Raposo, Polymer Eng. Sci. 330 (2009) 227. [6] G. Lagaly, Solid State Ionics 22 (1986) 43.

[7] H. S. Ferreira, Otimização do Processo de Organofilização de Bentonitas Visando seu Uso em Fluidos de Perfuração não Aquosos, Tese Dr. Eng. Processos, UFCG, Campina Grande, PB (2009).

[8] H. S. Ferreira, R. R. Menezes, H. S. Ferreira, A. B. Martins, G. A. Neves, H. C. Ferreira, Cerâmica 54, 329 (2008) 77.

[9] L. B. Paiva, A. R. Morales, F. R. Valenzuela Diaz, Appl. Clay Sci. 42 (2008) 8.

[10] R. Bernier, E. Garland, A. Glickman, F. Jones, H. Mairs, R. Melton, J. Ray, J. Smith, D. Thomas, J. Campbell, Int. Assoc. Oil \& Gas Producers Rept. 342, Londres, Inglaterra (2003).

[11] R. Caenn, G. V. Chillingar, J. Petroleum Sci. Eng. 14 (1996) 221.

[12] M. Kozak, L. Domka, J. Phys. Chem. Solids 65 (2004) 441.

[13] V. Mahto, V. P. Sharma, J. Petroleum Sci. Eng. 45 ( 2004) 123.

[14] Y. H. Shen, Chemosphere 44 (2001) 989.

[15] M. D. Foster, Am. Miner. 38 (1953) 994.

[16] Petrobras, Ensaio de argila organofilica para fluidos de perfuração à base de óleo, N-2259 (1997).

[17] L. V. Amorim, C. M. Gomes, H. L. Lira, K. B. França, H. C. Ferreira, Mater. Res. 7, 4 (2004) 583.

[18] R. R. Menezes, P. M. Souto, L. N. L. Santana, G. A. Neves, R. H. G. A. Kiminami, H. C. Ferreira, Cerâmica 55, 334 (2009) 163.

(Rec. 09/05/2011, Ac. 03/09/2011) 